\title{
lonic colloidal crystals of oppositely charged particles
}

\author{
Mirjam E. Leunissen ${ }^{1 \star}$, Christina G. Christova ${ }^{1 \star}$, Antti-Pekka Hynninen ${ }^{1}$, C. Patrick Royall ${ }^{1} \dagger$, Andrew I. Campbell ${ }^{1}$ \\ Arnout Imhof ${ }^{1}$, Marjolein Dijkstra ${ }^{1}$, René van Roij ${ }^{2} \&$ Alfons van Blaaderen $^{1}$
}

Colloidal suspensions are widely used to study processes such as melting, freezing ${ }^{1-3}$ and glass transitions $s^{4,5}$. This is because they display the same phase behaviour as atoms or molecules, with the nano- to micrometre size of the colloidal particles making it possible to observe them directly in real space ${ }^{3,4}$. Another attractive feature is that different types of colloidal interactions, such as long-range repulsive $e^{1,3}$, short-range attractive ${ }^{5}$, hard-spherelike $^{2-4}$ and dipolar ${ }^{3}$, can be realized and give rise to equilibrium phases. However, spherically symmetric, long-range attractions (that is, ionic interactions) have so far always resulted in irreversible colloidal aggregation ${ }^{6}$. Here we show that the electrostatic interaction between oppositely charged particles can be tuned such that large ionic colloidal crystals form readily, with our theory and simulations confirming the stability of these structures. We find that in contrast to atomic systems, the stoichiometry of our colloidal crystals is not dictated by charge neutrality; this allows us to obtain a remarkable diversity of new binary structures. An external electric field melts the crystals, confirming that the constituent particles are indeed oppositely charged. Colloidal model systems can thus be used to study the phase behaviour of ionic species. We also expect that our approach to controlling opposite-charge interactions will facilitate the production of binary crystals of micrometre-sized particles, which could find use as advanced materials for photonic applications ${ }^{7}$.

We recently developed a versatile colloidal model system of charged, sterically stabilized, polymethylmethacrylate (PMMA) spheres in a nearly density-matched mixture of cyclohexyl bromide $(\mathrm{CHB})$ and cis-decalin ${ }^{3,8}$. We simultaneously matched the refractive indices to eliminate the van der Waals forces. The range of the electrostatic interactions can be tuned with a salt like tetrabutylammonium bromide (TBAB) and is characterized by the Debye screening length, $\kappa^{-1}$, which for a number density $2 c$ of monovalent cations and anions is given by

$$
\kappa^{-1}=\left(8 \pi \lambda_{\mathrm{B}} c\right)^{-\frac{1}{2}}
$$

Here the Bjerrum length is $\lambda_{\mathrm{B}}=e^{2} /\left(4 \pi \varepsilon \varepsilon_{0} k_{\mathrm{B}} T\right)=10.0 \mathrm{~nm}$, with $\varepsilon$ the relative dielectric constant of our solvent, $\varepsilon_{0}$ the dielectric permittivity of vacuum, $e$ the elementary charge, $k_{\mathrm{B}}$ Boltzmann's constant and $T$ the absolute temperature. From measurements of the three-dimensional radial distribution function we found that these suspensions are well-described by a pair-wise screened Coulomb potential $V_{i j}(r)$ (ref. 8):

$$
\frac{V_{i j}(r)}{k_{\mathrm{B}} T}=Z_{i} Z_{j} \lambda_{\mathrm{B}} \frac{e^{\kappa\left(a_{i}+a_{j}\right)}}{\left(1+\kappa a_{i}\right)\left(1+\kappa a_{j}\right)} \frac{e^{-\kappa r}}{r}
$$

where $r$ is the distance between two particles with respective radii $a_{i / j}$ and charges $Z_{i / j}$.
Here we increase the complexity by investigating suspensions that consist of two types of colloids with opposite, dissimilar charges $Z_{1}$ and $Z_{2}$ and different radii $a_{1}$ and $a_{2}$. For binary systems, much less work is reported than for one-component suspensions and it mostly deals only with long-range repulsive $e^{9}$ and hard-sphere-like interactions $^{10,11}$ (although several researchers recently observed binary crystals of nanometre-sized colloids that probably formed in the presence of attractions ${ }^{12,13}$ ).

We discovered that in our apolar suspensions the addition of TBAB salt offers control over the particle charge, besides regulating the screening length. The charge even reverses from plus to minus at moderate salt concentrations ${ }^{8}$. Given the many components involved in the synthesis of the colloids ${ }^{14}$, it is not surprising that we obtain a slightly different charge reversal point for each batch of particles. Exploiting this, we prepared salt-containing mixtures of differently labelled fluorescent spheres, thereby establishing binary systems of colloids that carry small, opposite charges. Below, we present our observations on same-size spheres, followed by mixtures of large and small spheres.

For a relatively broad range of parameters we observed complete freezing into large, caesium-chloride-type $(\mathrm{CsCl})$ single crystals, that consist of several tens of particle layers measuring up to $300 \times 300 \mu \mathrm{m}$ (Supplementary Fig. 1). This even happens for colloids as large as the ones in Fig. 1a-d. These plus and minus particles had radii $a_{1}=1.08$ and $a_{2}=0.99 \mu \mathrm{m}$ respectively, that is, a size ratio $a_{2} / a_{1}$ close to unity (0.92). Typical suspensions with a $1: 1$ particle number ratio had an overall volume fraction $\varphi \approx 0.12$ and contained $\sim 60 \mu \mathrm{M}$ TBAB, corresponding to $\kappa^{-1}=285 \mathrm{~nm}$. From electrophoretic mobility measurements we found approximate charges of $Z_{1}=+110$ and $Z_{2}=-75$ ( \pm 10 , s.d.). As expected, for significantly higher charges only irreversible aggregation occurred. We stress that we did the measurements both with dilute suspensions of the individual particle species and in situ in the concentrated binary samples used for phase behaviour studies (see Methods section for details).

The $\mathrm{CsCl}$ character, that is, two interlaced simple cubic lattices together forming a body-centred cubic (b.c.c) lattice, was confirmed by quantitative confocal microscopy and by measuring the Bragg angles with light scattering (Supplementary Methods, Supplementary Table 1 and Supplementary Fig. 2). We found $2.36 \mu \mathrm{m}$ for the lattice parameter, which translates into a packing fraction of 0.71 . Within the experimental error, this is the closest CsCl-type packing possible for these spheres.

We followed the nucleation and growth of the crystals over time (Supplementary Fig. 3). Within $\sim 35$ h we see the first crystallites, their random orientation indicating homogeneous nucleation. This nucleation time is remarkably short for binary colloids, because slow 
diffusion and fast sedimentation were expected to hinder crystal growth for our $2-\mu \mathrm{m}$ spheres. Nevertheless, without fine-tuning the conditions, we observed significantly faster crystallization than, for instance, the four days reported for certain optimized hard-sphere systems of much smaller particles ${ }^{10,11}$. This ease of growth is important for the production of advanced materials, like photonic crystals, because bandgaps in the near-infrared require a dense, regular packing of micrometre-sized spheres ${ }^{7}$.

At $\varphi=0.30$ and $190 \mu \mathrm{M}$ TBAB $\left(\kappa^{-1}=195 \mathrm{~nm}\right)$ we observed a close-packed lattice with the plus and minus particles randomly distributed over the sites (Fig. 1e). This so-called 'solid solution' had a lattice spacing $\sim 6 \%$ larger than the densest possible packing (packing fraction 0.74 ). Semi-quantitative observations indicate that this different structure, which was recently predicted in simulations

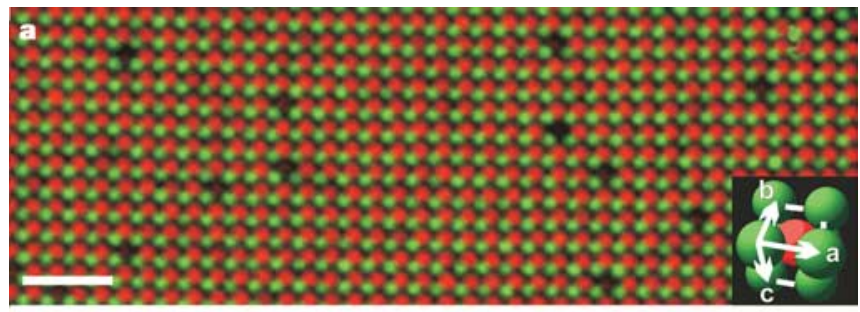

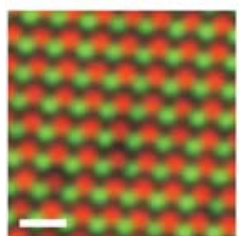
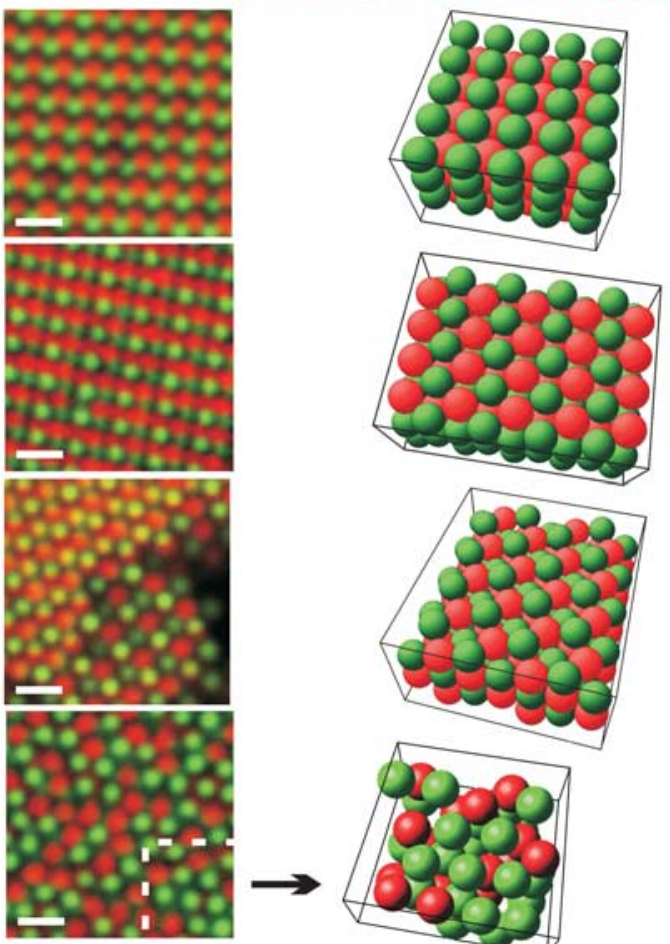

d
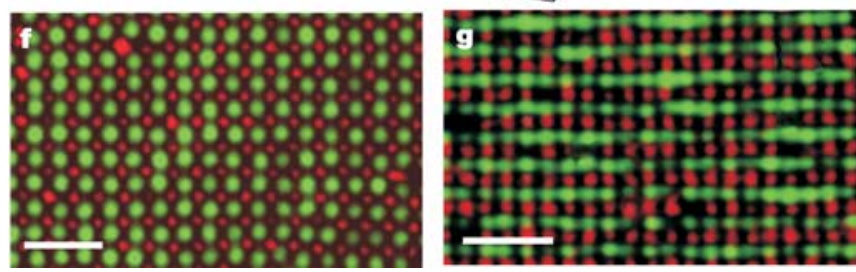

Figure 1 | CsCl-type binary crystals. a-e, Positive (red, radius $1.08 \mu \mathrm{m}$ ) and negative (green, $0.99 \mu \mathrm{m}$ ) PMMA-spheres. a, Confocal micrograph of a large (100) plane (scale bar, $10 \mu \mathrm{m}$ ). Inset, the cubic CsCl-type unit cell.

b-d, Close-up of the (100), (110) and (111) planes plus corresponding models. e, 'Solid solution'. The stacking of the hexagonal layers is visible in the box with rendered coordinates. The model spheres have a smaller radius for clarity. f, $\mathrm{CsCl}$ (100) and $\mathbf{g}$, (110) planes with positive PMMA (green, $0.52 \mu \mathrm{m}$ ) and negative silica (red, $0.58 \mu \mathrm{m}$ ) particles. Scale bars in $\mathbf{b}-\mathbf{g}, 4 \mu \mathrm{m}$. All particles were dispersed in TBAB-containing CHB-decalin. of the restricted primitive model ${ }^{15}$, is due to lower particle charges.

We also looked at binary crystals of two different materials, because the ability to create these is important for applications. We used PMMA ( $a_{1}=0.58 \mu \mathrm{m}$, positive) and silica $\left(a_{2}=0.52 \mu \mathrm{m}\right.$, negative) particles in a 53 vol. $\% / 47$ vol.\% CHB-decalin mixture ( $\varphi \approx 0.13-0.20$ and $\sim 160 \mu \mathrm{M}$ TBAB). Despite the large density contrast $\left(\sim 1.2 \mathrm{~g} \mathrm{~cm}^{-3}\right.$ for PMMA versus $2.0 \mathrm{~g} \mathrm{~cm}^{-3}$ for silica) the electrostatic attractions are strong enough to overcome differences in sedimentation. This produced quick growth of CsCl-type crystals again (Fig. 1f, g).

Previously, we demonstrated that PMMA can be burned away from composite crystals, leaving the silica spheres behind ${ }^{16}$. Using this procedure, one could turn a CsCl-type crystal into a simple cubic lattice, a structure that has not yet been realized by self-organization of colloids. Moreover, silica-only structures are important for the production of photonic crystals with 'inverse' index contrast, as this often requires a high temperature reaction ${ }^{7}$.

We confirmed that the particles inside the binary crystals are still oppositely charged by applying an external, static electric field. Figure 2a shows how a CsCl-type crystal melts as the differently labelled particles move towards opposite electrodes. This also demonstrates
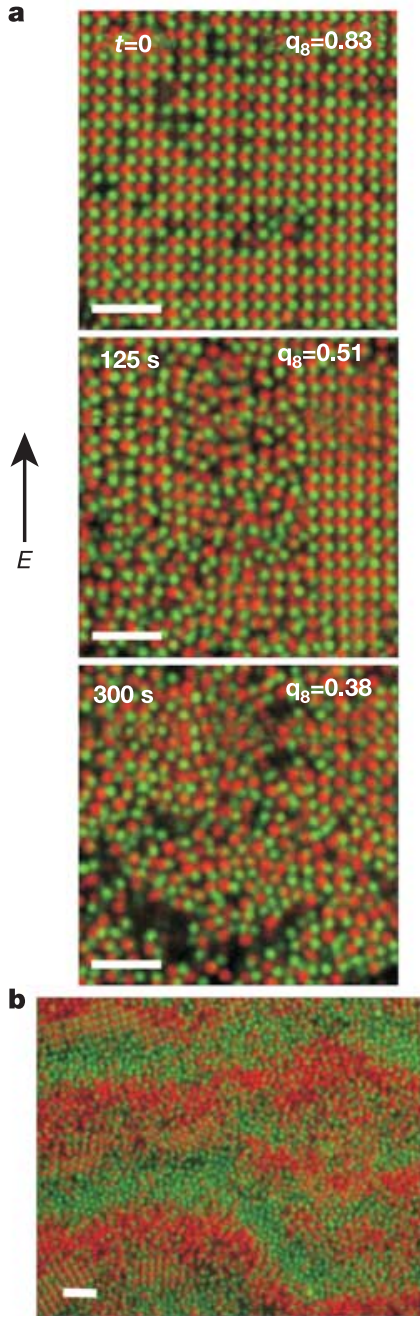
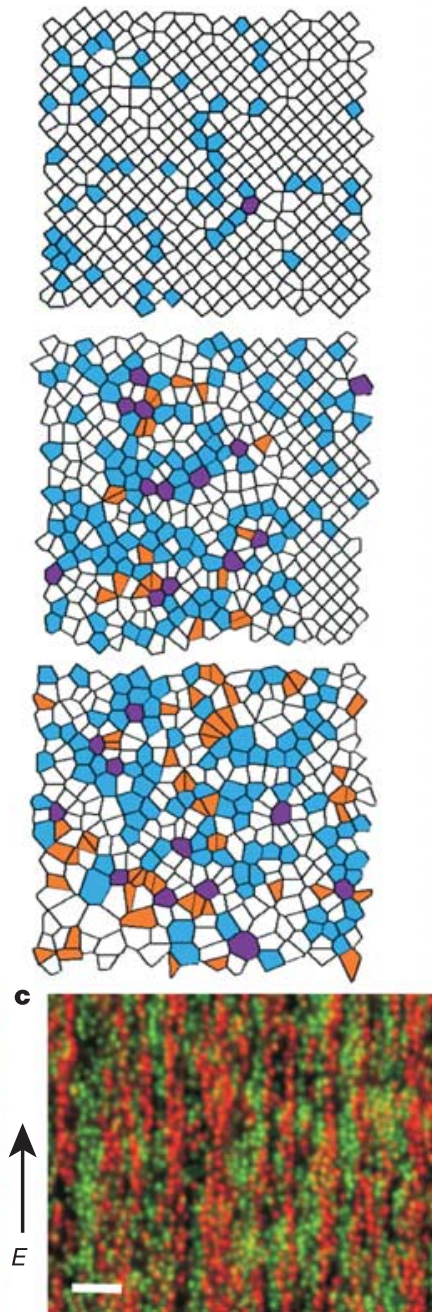

Figure 2 | Electric-field-induced melting. a, Confocal snapshots of the melting process induced by a static electric field $\left(E=7 \mathrm{~V} \mathrm{~mm}^{-1}\right)$ at $t=0$, 125 and $300 \mathrm{~s}$. The frame-averaged value of the bond order parameter $q_{8}$ is indicated. In the Voronoi constructions orange polygons are threefoldcoordinated; white, fourfold; blue, fivefold; purple, sixfold ${ }^{31}$. b, Jammed 'band' formation in a dense sample. c, Dynamic lane formation at lower density and $80 \mathrm{~V} \mathrm{~mm}^{-1}$. All scale bars are $10 \mu \mathrm{m}$. 
that electric fields are a powerful way to manipulate these soft colloidal crystals. Here, a mere $7 \mathrm{~V} \mathrm{~mm}^{-1}$ sufficed. Furthermore, we note that this system is very similar to a certain electronic ink ('e-ink') ${ }^{17}$, although e-ink aggregates.

In some cases the electric field induced interesting pattern formation. Dense suspensions $(\varphi>0.40)$ in fairly high fields $\left(\sim 20 \mathrm{~V} \mathrm{~mm}^{-1}\right)$ form 'bands' perpendicular to the field direction (Fig. 2b). The different particles initially move in opposite directions, but soon form a glassy state, which no longer shows any diffusion or field-induced drift. This process is reversible: the system 'un-jams' when the field is switched off. In relatively dilute suspensions $(\varphi \approx 0.20-0.30)$ the colloids remain mobile and another pattern appears, with the oppositely driven particles segregated into 'lanes' along the field direction (Fig. 2c). This so-called 'lane formation' is a first-order out-of-equilibrium phase transition ${ }^{18,19}$. It can now be studied in a controlled way and quantitatively at the single particle level.

We also investigated mixtures of large (L) and small (S) spheres, with a considerable size difference $\left(a_{2} / a_{1}=0.31\right)$. Using a $1: 8$ (L:S number density) suspension of negative $\left(a_{1}=1.16 \mu \mathrm{m}\right)$ and positive $\left(a_{2}=0.36 \mu \mathrm{m}\right)$ PMMA-particles $(\varphi \approx 0.11$, estimated TBAB-concentration $\sim 120 \mu \mathrm{M})$ we observed crystals with $\mathrm{LS}_{6}$ stoichiometry (Fig. 3). This new structure has a face-centred orthorhombic lattice of the large spheres. One small sphere occupies each of the tetrahedral holes, while four small spheres are found in each of the octahedral holes (two slightly above and two slightly below each $a b$-plane of large particles in Fig. $3 a$ and $d$ ). The remaining small spheres are expelled from the crystal. (After submission of this Letter Pusey et al. drew our attention to their observations of a slightly different colloidal $\mathrm{LS}_{6}$-structure, which has a b.c.c. symmetry ${ }^{20}$. However, this was reported to be a binary hard-sphere mixture and no further details were given. We speculate that these binary crystals were also grown from oppositely charged colloids). Interestingly, these colloidal structures resemble the crystals that are formed by
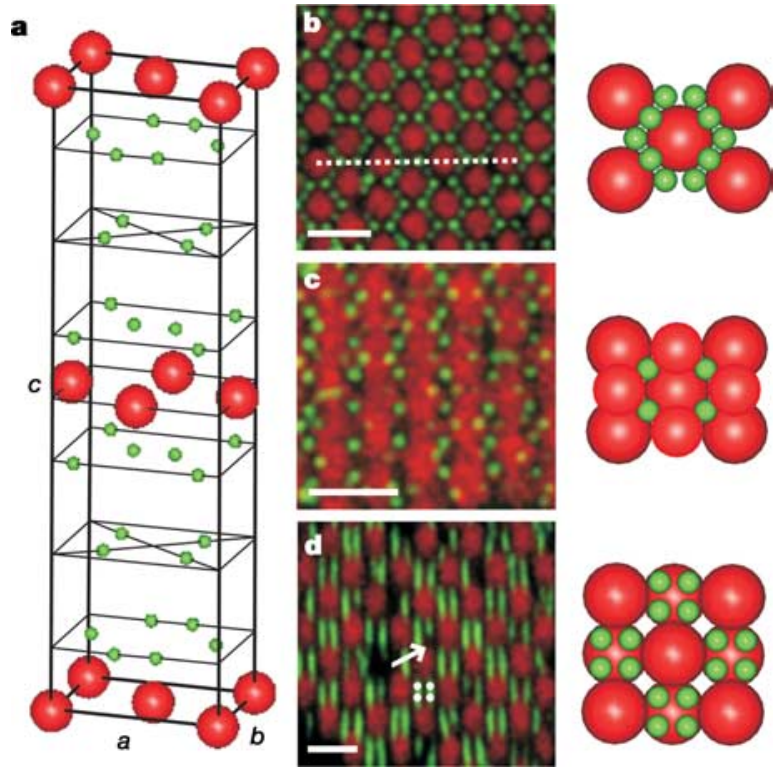

Figure 3 | LS $_{\mathbf{6}}$-type binary crystals. a-d, Positive (green, radius $0.36 \mu \mathrm{m}$ ) and negative (red, $1.16 \mu \mathrm{m}$ ) PMMA-particles in TBAB-containing CHBdecalin, forming a structure with $\mathrm{LS}_{6}$ stoichiometry. a, Unit cell with observed lattice spacings $a=4.00, b=2.84$ and $c=4.7 \mu \mathrm{m}$ (not to scale for clarity). b, c, Confocal images and models of different $a b$-projections, showing a layer of large and several layers of small particles (b) and a plane with only small particles (c). d, ac-cut along the line in $\mathbf{b}$. As the microscope could not completely resolve the four small particles in each octahedral hole, we indicated their positions with dots. The arrow indicates a missing particle. All scale bars are $4 \mu \mathrm{m}$. certain alkali-metal intercalation compounds of the fullerene $\mathrm{C}_{60}$ with the same stoichiometry ${ }^{21}$. In these compounds there is a large size difference between the constituent ions too.

At slightly lower ionic strength we found another new structure, $\mathrm{LS}_{8}$, for which no atomic or molecular counterpart has yet been identified. In this case, each large colloid is surrounded by six small spheres that occupy the trigonal interstices of the hexagonal planes. Above and below this 'mixed' layer are planes of small particles only in a Kagomé-type arrangement. This three-plane unit repeats itself in such a way that the large colloids form an $A B A B$-stacking.

It also proved possible to establish conditions under which the small spheres were essentially uncharged, while the large spheres were slightly charged, as judged from their electrophoretic mobility and the lattice spacing in crystals of the individual particle species. In a $\mathrm{L}: \mathrm{S}=1: 4$ suspension with $\varphi \approx 0.23$ and no added salt
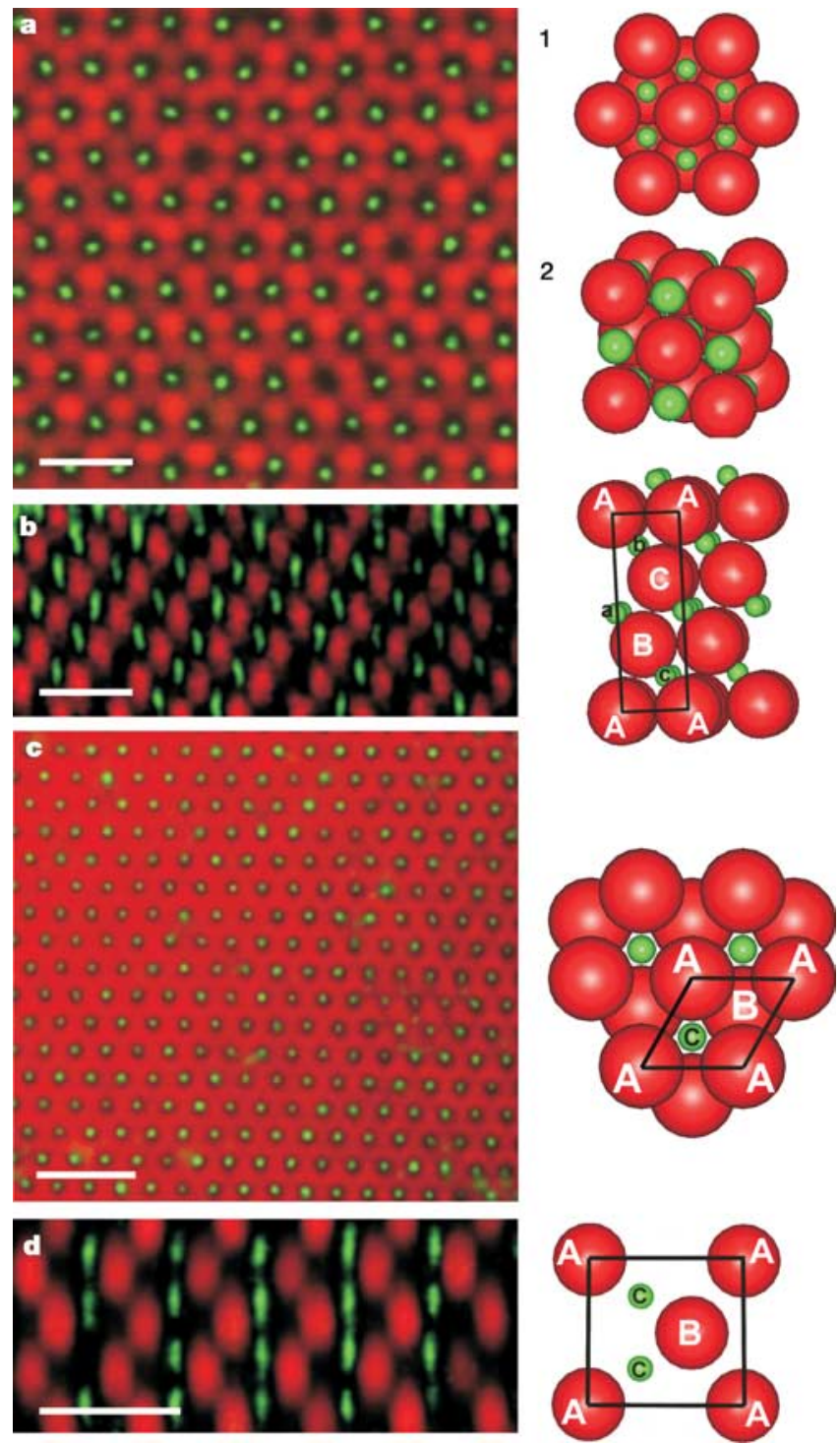

Figure 4 | LS-type binary crystals. Charged (red, radius $1.16 \mu \mathrm{m}$ ) and uncharged (green, $0.36 \mu \mathrm{m}$ ) PMMA-particles in CHB-decalin. a, b, NaCltype crystal. a, Confocal image of the hexagonal plane and the unit cell in an hexagonal (1) and a cubic (2) representation (small spheres enlarged for clarity). b. Plane perpendicular to the hexagonal close packed layers, showing the $A B C$-stacking of both the large and small particles. c, d, NiAstype crystal. c, Superposition of confocal images of ten layers and the corresponding model. $\mathbf{d}$, Plane as in $\mathbf{b}$, the model shows the $A B A$-stacking of the large and the $c c c$-stacking of the small particles (spheres not to scale). All scale bars are $8 \mu \mathrm{m}$. 
$\left(\kappa^{-1}=1.57 \mu \mathrm{m}\right)$ we observed coexistence of two different crystal structures, both with LS stoichiometry: sodium chloride type $(\mathrm{NaCl})$ and nickel arsenide type (NiAs) (Fig. 4). The latter structure has a hexagonal-close-packed (h.c.p.) stacking of the large spheres with all the octahedral sites occupied by the small spheres, which form a simple hexagonal sublattice. In the $\mathrm{NaCl}$-type structure the large spheres form an face-centred cubic (f.c.c.) lattice. The small spheres fill the octahedral holes again, but now as an interpenetrating f.c.c. lattice. Recently, LS-type structures were also observed in hard-sphere suspensions ${ }^{11}$ and in systems of nanocrystals with attractions ${ }^{12}$.

Although ionic crystals have been theoretically investigated in many papers, we found only one $\mathrm{e}^{22}$ that deals with oppositely charged spheres interacting through a screened Coulomb potential. (After submitting this Letter we became aware of a thesis ${ }^{23}$ that contains
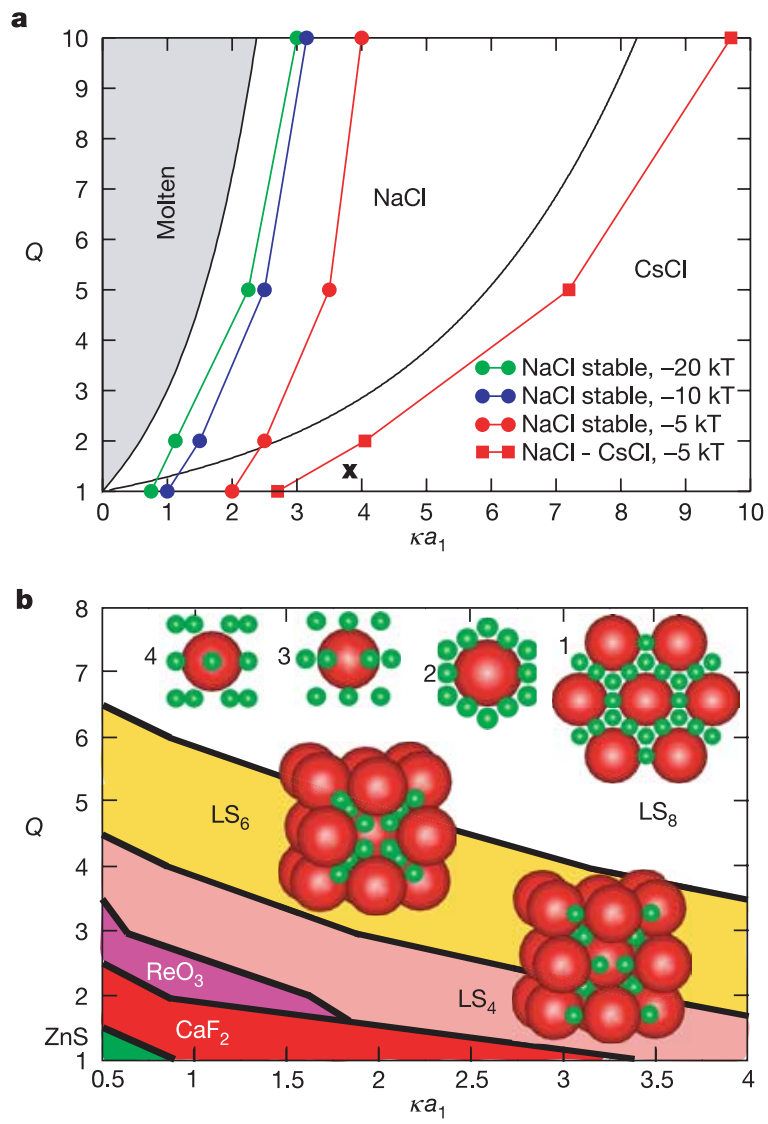

Figure 5 | Theoretical phase diagrams. Based on screened-Coulomb pairinteractions (equation (2)) for different screening constant, $\kappa a_{1}$, and charge ratio $Q=-Z_{1} / Z_{2}$ at a size ratio $a_{2} / a_{1}$ of 1 (a) and $0.31(\mathbf{b})$. Full curves indicate crystal-crystal transitions from Madelung energy calculations (zero-temperature; black) and finite-temperature, zero-pressure MC-simulations with a contact energy $V_{12}$ of $-20 k_{\mathrm{B}} T$ (green), $-10 k_{\mathrm{B}} T$ (blue) and $-5 k_{\mathrm{B}} T$ (red). a, The NaCl-structure melting line and $\mathrm{NaCl}-\mathrm{CsCl}$ phase boundary. The cross indicates the estimated state point of the experimentally observed $\mathrm{CsCl}$ crystals (Fig. 1a-d). NiAs, $\mathrm{CuAu}$ and $\mathrm{ZnS}$ structures, which we also evaluated, are un- or metastable. b, Crystal phases coexisting with a dilute gas of small colloids, from Madelung energies.

Unlike $\mathrm{LS}_{6}$ and $\mathrm{LS}_{8}$ the $\mathrm{LS}_{4}$ structure was not experimentally observed, but generated by removing two small particles from the octahedral holes of $\mathrm{LS}_{6}$ (compare the small colloid configuration around the central large sphere in the two models). In the $\mathrm{LS}_{8}$ structure a unit of three particle planes (insets 3 , 4 ; not to scale) repeats itself to form a hexagonal $A B A B$-stacking of the large colloids. Insets: 1 , projection of the three basic planes of $\mathrm{LS}_{8}, 2$, configuration around a large colloid, 3 , side-view, 4 , side-view rotated by 90 degrees. We also evaluated structures of $\mathrm{NaCl}, \mathrm{CsCl}, \mathrm{NiAs}, \mathrm{CuAu}, \mathrm{AlB}_{2}, \mathrm{Cu}_{3} \mathrm{Au}, \mathrm{Al}_{3} \mathrm{Ti}$ and $\mathrm{CaCu}_{5}$, but these were found to be unstable. theoretical investigations and computer simulations of the interactions between oppositely charged colloids and experimental attempts to realise binary crystals of such particles.) Unfortunately, it restricts itself to a single set of interaction parameters, so we explored the stability of different crystal structures further ourselves. We did this for the size ratios studied experimentally $(\sim 1$ and 0.31$)$, as a function of the charge ratio $Q=-Z_{1} / Z_{2}$ and the reduced screening constant $\kappa a_{1}$.

For long screening lengths (equation (1)) our oppositely charged particles resemble ionic systems, but with differences. Most importantly, colloids together with their surrounding 'diffuse layer' of counter-ions are charge-neutral objects. Therefore, the stoichiometry is not dictated by charge neutrality: even with a considerable charge asymmetry colloids can form crystals with LS (or some other) stoichiometry, as in Fig. 1.

The equilibrium structure of a crystal is determined by geometric requirements, stemming from short-range repulsions, together with the potential energy of the lattice and the entropy associated with thermal effects. For ionic systems, the potential energy strongly depends on the Coulomb interactions, which we describe by the pair-potential of equation (2). This probably is a good approximation for the like-charge repulsions ${ }^{8}$, but its accuracy for the plusminus attractions is not well known (see below). We further assume a vanishingly small osmotic pressure, such that the crystals are selfsupported by their cohesive energy and coexist with a zero-density vapour.

The phase diagrams in Fig. 5 display structures with minimal Madelung energy (potential energy per particle in the perfect crystal; we assume touching neighbours), in which we neglect entropy. In this zero-temperature limit the phase diagram depends on the charge ratio and not on the absolute contact energies $V_{i j}$ (equation (2) evaluated at $r=a_{i}+a_{j}$ ). The absolute charges only become relevant in the Monte Carlo simulations that we performed in order to assess the importance of entropy at ambient temperature (see Methods section).

The experimentally determined charges (of the particles in Fig. 1a-d) give a contact energy $V_{12}$ of $\sim-2 k_{\mathrm{B}} T$ for the plusminus interactions. However, we bear in mind that the electric field gradient becomes very steep near contact. It is not known whether the Poisson-Boltzmann approach underlying equation (2) is valid in this case, nor what is the correct boundary condition (that is, constant surface charge versus potential or something in between). Therefore, we chose several contact energies and examined how this affected the stability lines.

Figure $5 \mathrm{a}$ shows that the $\mathrm{NaCl}$ and $\mathrm{CsCl}$ structures dominate for same-size colloids. If we include entropic effects, both the $\mathrm{NaCl}-\mathrm{CsCl}$ phase boundary and the $\mathrm{NaCl}$ melting line shift to higher $\kappa a_{1}$ (more screening). In our experiments, the clear particle motion around the lattice positions indicates that entropy is indeed important. The observed CsCl-type crystals (Fig. 1a-d) lie inside the predicted $\mathrm{CsCl}$ regime. Their Madelung energy was calculated to be $\sim-6 k_{\mathrm{B}} T$ (per particle). In the 'molten' regime (positive $\mathrm{NaCl}$ Madelung energy) we find many other crystal phases, but this will be discussed elsewhere.

The phase diagram for size ratio 0.31 (Fig. 5b) nicely demonstrates how the stoichiometry follows the charge ratio at low $\kappa a_{1}$. We obtained this diagram with the common tangent construction, taking the dilute phase rich in small colloids. Of course, this is just one of many possible 'slices' through the multidimensional phase diagram. If we choose the composition of the dilute phase differently, we obtain a different diagram. However, the main features, such as the order of the phases along the $Q$-axis, remain the same. Thus, the charge ratio in the experimentally observed $\mathrm{LS}_{6}$-structure is probably smaller than six. The diagram also contains a region where the $\mathrm{LS}_{8}$-structure is stable. The insets in Fig. $5 \mathrm{~b}$ show a model of this structure; we will present experimental data elsewhere.

Our present experiments and those of others demonstrate that 
equilibrium phase behaviour of oppositely charged colloids occurs for a fairly broad range of parameters and we expect that binary crystals should be observable for particle sizes covering the entire colloidal range. Evidence of crystallizing plus and minus spheres has been observed under conditions different from those used here ${ }^{24}$. We also suspect that Underwood et al..$^{25}$ may have ruled out the presence of opposite charges too quickly as the origin of the attractions that caused their CsCl-type crystals. All of these studies involved organic solvents, but we expect that ionic crystals can also be obtained in more polar solvents, such as water. Although it is more difficult to keep the van der Waals forces sufficiently small in water, this was recently achieved for a suspension with attractive interactions mediated through complementary strands of DNA on the particles ${ }^{26}$. A mixture with different complementary sequences for different particles would actually mimic our system for short screening lengths.

\section{METHODS}

Particle synthesis. We used polymethylmethacrylate (PMMA) and silica particles. The former were made by dispersion polymerization, covalently labelled with the fluorophore 7-nitrobenzo-2-oxa-1,3-diazol (NBD) or rhodamine isothiocyanate (RITC) and sterically stabilized with poly-12-hydroxystearic acid (PHSA) ${ }^{14}$. These particles had a size polydispersity of 3-5\% (from light scattering and electron microscopy). We prepared the silica colloids using a modified Stöber synthesis ${ }^{27}$, which includes continuous-feed seeded growth, resulting in an RITC-labelled core of radius $200 \mathrm{~nm}$ surrounded by an unlabelled shell. The particles were then coated with 3-methacryloxypropyltrimethoxysilane (TPM) and stabilized with PHSA. The total radius was $0.58 \mu \mathrm{m}$, with $4 \%$ polydispersity.

Sample preparation. Unless indicated otherwise, the colloids were dispersed in a mixture of cyclohexyl bromide (CHB, Fluka) and $27.2 \mathrm{wt} \%$ cis-decalin (SigmaAldrich) that nearly matches the density and refractive index of PMMA. We purified the CHB as described before ${ }^{8}$, but skipped the distillation. The particle charge and the range of the electrostatic interactions were tuned by adding tetrabutylammonium bromide salt (TBAB, Sigma-Aldrich) ${ }^{8}$. We let separate suspensions of the individual particle species equilibrate for several hours before mixing them together. We then confined the binary mixture to glass capillaries (VitroCom) and studied it by means of confocal laser scanning microscopy, extracting the three-dimensional particle coordinates as described before ${ }^{8}$. Gradient samples served to explore the phase behaviour as a function of the salt concentration. We made these by filling a capillary partly with a salt-free suspension and partly with a TBAB solution and allowing it to form macroscopic gradients in a few days' time (at constant particle volume fraction).

Electrical conductivity measurements. We estimated the Debye screening length of our suspensions by measuring the conductivity of the particle-free solvent mixtures (with a Scientifica 627 conductivity meter) and then applying Walden's rule ${ }^{8}$. We used literature values for the limiting equivalent conductance of TBAB in water and a viscosity of 2.217 centipoise at $25^{\circ} \mathrm{C}$ (measured with a Schott ViscoSystem) for the solvent mixture.

Electrophoretic mobility measurements. Particle charges were quantified by means of electrophoresis (using a Coulter Delsa 440SX) on dilute suspensions (volume fraction 0.0015 ) in the $\mathrm{CHB}$-decalin mixture ${ }^{8}$. The run parameters were: $25 \mathrm{~V}, 2.0 \mathrm{~s}$ on $/ 0.5 \mathrm{~s}$ off, and a total run time of $60 \mathrm{~s}$. We identified the stationary layers by Komagata linearization ${ }^{28}$ and related the electrophoretic mobility to the zeta potential via the O'Brien and White scheme ${ }^{29}$. From dielectric spectroscopy (with an HP 4294A impedance analyser) the dielectric constant of the solvent mixture was found to be 5.59.

We also estimated the charges in the denser microscopy samples. The sample cell consisted of a $0.1 \times 1 \mathrm{~mm}$ capillary with two $50-\mu \mathrm{m}$-diameter nickel-alloy wires (Goodfellow) threaded through along the side walls. We applied a directcurrent field (up to $25 \mathrm{~V} \mathrm{~mm}^{-1}$ ) and captured the electrophoretic motion of individual particles shortly after they left the binary crystals (scan speed 1-3 frames/second). We determined the particles' mobility from the displacements between subsequent frames.

Theory and computer simulations. We parameterized our Madelung energy calculations and computer simulations by choosing values for the reduced screening constant $\kappa a_{1}$, the charge ratio $Q=-Z_{1} / Z_{2}$ and the contact energy $V_{12}\left(a_{1}+a_{2}\right)$. These parameters, together with the size ratio $a_{2} / a_{1}$, fully determine the pair potential of equation (2). We performed so-called 'constant-NPT' Monte Carlo simulations, thus keeping the number of particles $N$, the pressure $P$ and the temperature $T$ fixed. If a crystal coexists with a lowdensity gas one can estimate its coexistence density with a simulation at zero- pressure $^{30}$. In this way we obtained the NaCl-structure melting lines and the stabilities of all other crystal structures. The $\mathrm{NaCl}-\mathrm{CsCl}$ phase line at a contact energy $V_{12}$ of $-5 k_{\mathrm{B}} T$ was determined by first minimizing the free energy of both phases with respect to density and then finding the point where the free energy per particle of the two phases is equal. We calculated the free energies from 'constant-NVT' Monte Carlo simulations (fixing the number of particles $N$, the volume $V$ and the temperature $T$ ) using the Frenkel-Ladd method ${ }^{30}$. We did all the simulations in a cubic box with periodic boundary conditions, taking 216 and 250 particles for the $\mathrm{NaCl}$ - and $\mathrm{CsCl}$-crystals respectively.

We determined the Madelung energy with the potential energy calculation in the Monte Carlo code, assuming contact between neighbouring spheres in a structure. The absolute value of $V_{12}$ does not play a role as long as the same value is used throughout. For the $\mathrm{LS}_{4}, \mathrm{LS}_{6}$ and $\mathrm{LS}_{8}$ structures the energy minimum was found by varying their lattice parameters. We then drew up the phase diagram with the common tangent construction, taking the dilute phase rich in small colloids. All the other structures that we evaluated can be found in the legend of Fig. 5.

\section{Received 11 April; accepted 20 June 2005}

1. Hachisu, S., Kobayashi, Y. \& Kose, A. Phase separation in monodisperse latexes. J. Colloid Interf. Sci. 42, 342-348 (1973).

2. Pusey, P. N. \& van Megen, W. Phase behaviour of concentrated suspensions of nearly hard colloidal spheres. Nature 320, 340-342 (1986).

3. Yethiraj, A. \& van Blaaderen, A. A colloidal model system with an interaction tunable from hard sphere to soft and dipolar. Nature 421, 513-517 (2003).

4. Kegel, W. K. \& van Blaaderen, A. Direct observation of dynamical heterogeneities in colloidal hard-sphere suspensions. Science 287, 290-293 (2000).

5. Pham, K. N. et al. Multiple glassy states in a simple model system. Science 296, 104-106 (2002).

6. Islam, A. M., Chowdhry, B. Z. \& Snowden, M. J. Heteroaggregation in colloidal dispersions. Adv. Colloid Interf. 62, 109-136 (1995).

7. Vlasov, Y. A., Bo, X. Z., Sturm, J. C. \& Norris, D. J. On-chip natural assembly of silicon photonic bandgap crystals. Nature 414, 289-293 (2001)

8. Royall, C. P., Leunissen, M. E. \& van Blaaderen, A. A new colloidal model system to study long-range interactions quantitatively in real space. J. Phys. Condens. Matter 15, S3581-S3596 (2003).

9. Hachisu, S. \& Yoshimura, S. in Physics of Complex and Supermolecular Fluids (eds Safran, S. A. \& Clark, N. A.) 221-240 (Wiley, New York, 1987).

10. Schofield, A. B. Binary hard-sphere crystals with the cesium chloride structure. Phys. Rev. E 64, 051403 (2001)

11. Hunt, N., Jardine, R. \& Bartlett, P. Superlattice formation in mixtures of hardsphere colloids. Phys. Rev. E 62, 900-913 (2000).

12. Saunders, A. E. \& Korgel, B. A. Observation of an AB phase in bidisperse nanocrystal superlattices. Chemphyschem 6, 61-65 (2005).

13. Redl, F. X., Cho, K. S., Murray, C. B. \& O'Brien, S. Three-dimensional binary superlattices of magnetic nanocrystals and semiconductor quantum dots. Nature 423, 968-971 (2003).

14. Bosma, G. et al. Preparation of monodisperse, fluorescent PMMA-latex colloids by dispersion polymerization. J. Colloid Interf. Sci. 245, 292-300 (2002).

15. Bresme, F., Vega, C. \& Abascal, J. L. F. Order-disorder transition in the solid phase of a charged hard sphere model. Phys. Rev. Lett. 85, 3217-3220 (2000)

16. Velikov, K. P., Christova, C. G., Dullens, R. P. A. \& van Blaaderen, A. Layer-bylayer growth of binary colloidal crystals. Science 296, 106-109 (2002).

17. Gelinck, G. H. et al. Flexible active-matrix displays and shift registers based on solution-processed organic transistors. Nature Mater. 3, 106-110 (2004).

18. Dzubiella, J., Hoffmann, G. P. \& Löwen, H. Lane formation in colloidal mixtures driven by an external field. Phys. Rev. E 65, 021402 (2002)

19. Löwen, H. \& Dzubiella, J. Nonequilibrium pattern formation in strongly interacting driven colloids. Faraday Discuss. 123, 99-105 (2003).

20. Pusey, P. N. General discussion. Faraday Discuss. 123, 177 (2003)

21. Dresselhaus, M. S., Dresselhaus, G. \& Eklund, P. C. Science of Fullerenes and Carbon Nanotubes Ch. 8 (Academic, London, 1996).

22. Caballero, J. B., Puertas, A. M., Fernandez-Barbero, A. \& de las Nieves, F. J. Oppositely charged colloidal binary mixtures: A colloidal analog of the restricted primitive model. J. Chem. Phys. 121, 2428-2435 (2004).

23. Maskaly, G. R. Attractive Electrostatic Self-Assembly of Ordered and Disordered Heterogeneous Colloids. PhD thesis, Massachusetts Institute of Technology (2005)

24. Bartlett, P. \& Campbell, A. I. Three-dimensional binary superlattices of oppositely charged colloids. Phys. Rev. Lett. (in the press).

25. Underwood, S. M., van Megen, W. \& Pusey, P. N. Observation of colloidal crystals with the cesium-chloride structure. Physica A 221, 438-444 (1995).

26. Biancaniello, P. L., Kim, A. J. \& Crocker, J. C. Colloidal interactions and selfassembly using DNA hybridization. Phys. Rev. Lett. 94, 058302 (2005).

27. van Blaaderen, A. \& Vrij, A. Synthesis and characterization of colloidal dispersions of fluorescent, monodisperse silica spheres. Langmuir 8, 2921-2931 (1992) 
28. Hunter, R. J. Zeta Potential in Colloid Science Ch. 4 (Academic, London, 1981)

29. O'Brien, R. W. \& White, L. R. Electrophoretic mobility of a spherical colloidal particle. J. Chem. Soc. Faraday Trans. II 74, 1607-1626 (1978).

30. Frenkel, D. \& Smit, B. Understanding Molecular Simulations Ch. 5, 10 (Academic, London, 2002).

31. Weiss, J. A., Oxtoby, D. W., Grier, D. G. \& Murray, C. A. Martensitic transition in a confined colloidal suspension. J. Chem. Phys. 103, 1180-1190 (1995).

Supplementary Information is linked to the online version of the paper at www.nature.com/nature.

Acknowledgements We thank R. P. A. Dullens, D. Derks, N. A. M. Verhaegh, P. Vergeer and C. M. van Kats for particle synthesis, A. D. Hollingsworth for solvent characterization, J. H. J. Thijssen for help with the pictures of the Bragg reflections and both P.N. Pusey and A.B. Schofield for pointing out the resemblance between our $\mathrm{LS}_{6}$-structure and certain fullerene compounds. This work is part of the research program of the 'Stichting voor Fundamenteel Onderzoek der Materie' (FOM), which is financially supported by the 'Nederlandse organisatie voor Wetenschappelijk Onderzoek' (NWO).

Author contributions M.E.L. and C.G.C. investigated the phase behaviour of the experimental binary systems, A.-P.H. and M.D. performed the computer simulations, A.-P.H. and R.v.R. calculated the Madelung energies, C.P.R. worked on the lane formation, A.I. on the Bragg scattering, A.I.C. made different plus/ minus systems and A.v.B. initiated the work and co-wrote the paper together with M.E.L.

Author Information Reprints and permissions information is available at npg.nature.com/reprintsandpermissions. The authors declare no competing financial interests. Correspondence and requests for materials should be addressed to M.E.L. (M.E.Leunissen@phys.uu.nl) or A.v.B.

(A.vanBlaaderen@phys.uu.nl). 